\title{
Medication therapy management and adherence among US renal transplant recipients
}

\author{
This article was published in the following Dove Press journal: \\ Patient Preference and Adherence \\ 28 April 2016 \\ Number of times this article has been viewed
}

\author{
Marie A Chisholm-Burns ${ }^{1}$ \\ Christina A Spivey' \\ Elizabeth A Tolley ${ }^{2}$ \\ Erin K Kaplan² \\ 'Department of Clinical Pharmacy, \\ University of Tennessee College of \\ Pharmacy, ${ }^{2}$ Department of Preventive \\ Medicine, University of Tennessee \\ College of Medicine, Memphis, \\ TN, USA
}

\begin{abstract}
Background: Medication therapy management (MTM) services among patient populations with a range of disease states have improved adherence rates. However, no published studies have examined the impact of Medicare Part D MTM eligibility on renal transplant recipients' (RTRs) immunosuppressant therapy (IST) adherence. This study's purpose was therefore, to determine the effects of Medicare Part D MTM on IST adherence among adult RTRs at 12 months posttransplant.
\end{abstract}

Methods: Cross-sectional analyses were performed on Medicare Parts A, B, and D claims and transplant follow-up data reported in the United States Renal Data System. The sample included adult RTRs who were transplanted between 2006 and 2011, had graft survival for 12 months, were enrolled in Part D, and were prescribed tacrolimus. IST adherence was measured by medication possession ratio for tacrolimus. MTM eligibility was determined using criteria established by the Centers for Medicare and Medicaid Services. Descriptive statistics were calculated. Adherence was modeled using multiple logistic regression.

Results: In all, 17,181 RTRs were included. The majority of the sample were male (59.1\%), and $42 \%$ were MTM-eligible. Mean medication possession ratio was $0.91 \pm 0.17$ (mean \pm standard deviation), with $16.83 \%$ having a medication possession ratio of $<0.80$. MTM eligibility, sex, age, and number of prescription drugs were significantly associated with adherence in the full model $(P<0.05)$. MTM-eligible RTRs were more likely to be adherent than those who were not MTM-eligible (odds ratio $=1.13,95 \%$ confidence interval $1.02-1.26, P=0.02$ ).

Conclusion: The findings provide evidence that access to MTM services increases IST adherence among RTRs.

Keywords: Medicare Part D, medication adherence, medication therapy management, renal transplant

\section{Introduction}

Among renal transplant recipients (RTRs), adherence to immunosuppressant therapy (IST) is considered critical to graft survival. ${ }^{1,2}$ Yet, rates of IST nonadherence are variable, ranging from $<20 \%$ to $>40 \%{ }^{1-4}$ Numerous barriers to IST adherence exist and include, but are not limited to, drug cost, confusion about the IST regimen, polypharmacy (as the RTR's regimen may include IST as well as medications used to treat comorbid conditions), and psychosocial factors. ${ }^{4,5}$ To assist in addressing drug cost barriers, RTRs who qualify for Medicare Part B due to end-stage renal disease receive 36 months of coverage for a portion of their IST costs; those RTRs who qualify for Medicare due to age or disability are eligible for lifetime coverage. However, nonadherence persists even when cost is reduced as a barrier, suggesting additional interventions are needed. ${ }^{6}$ Medication therapy management (MTM) is one such intervention that has demonstrated success in improving medication adherence and patient outcomes in chronic disease patients. ${ }^{7}$
Correspondence: Marie A Chisholm-Burns Department of Clinical Pharmacy, University of Tennessee College of Pharmacy, 88I Madison Ave., Ste 264, Memphis, TN 38163, USA

$\mathrm{Tel}+\mathrm{I}$ 90I 4486036

Email mchisho3@uthsc.edu (c) (i) (c) 2016 Chisholm-Burns et al. This work is published and licensed by Dove Medical Press Limited. The full terms of this license are available at https://www.dovepress.com/terms.php cc) ${ }_{\mathrm{BY}} \mathrm{NC}$ and incorporate the Creative Commons Attribution - Non Commercial (unported, v3.0) License (http://creativecommons.org/licenses/by-nc/3.0/). By accessing the work you hereby accept the Terms. Non-commercial uses of the work are permitted without any further permission from Dove Medical Press Limited, provided the work is properly attributed. For permission for commercial use of this work, please see paragraphs 4.2 and 5 of our Terms (https://www.dovepress.com/terms.php). 
In 2006, the Medicare Part D program was enacted to provide prescription drug coverage to Medicare beneficiaries. MTM is a benefit of the Part D program available to those beneficiaries, including RTRs with Medicare coverage, who meet criteria established by the Centers for Medicare and Medicaid Services (CMS). ${ }^{8}$ According to the American Pharmacists Association, the purpose of MTM is to "optimize therapeutic outcomes for individual patients." Components include performing a medication therapy review, developing a medication treatment plan, providing education related to the medication regimen and disease state, and monitoring patient response to medication therapy. ${ }^{10}$

A systematic review found that outpatient MTM services among patient populations with a range of disease states have improved adherence rates, percent of patients reaching a threshold adherence level, and medication appropriateness. ${ }^{7}$ MTM services have also been associated with other benefits, such as reduced health care costs, increased institutional cost savings, and improved surrogate clinical markers (eg, increased blood pressure control, decreased cholesterol levels). ${ }^{11-14}$ However, a search of the literature (PubMed, years unlimited) revealed that no published studies have examined the impact of Part D MTM eligibility on RTRs' IST adherence. Therefore, the objective of this study was to determine the effect of Medicare Part D MTM eligibility on IST adherence among adult RTRs at 12 months posttransplant, a critical time period in establishing desirable medication-taking behavior patterns.

\section{Materials and methods}

Cross-sectional analyses were performed on Medicare Parts A, B, and D claims data and follow-up data reported to the United Network for Organ Sharing database in the United States Renal Data System (USRDS). United Network for Organ Sharing transplant center members are required to submit information on RTRs using standardized data collection forms at the time of transplant candidate registration, transplant, 6 months posttransplant, and transplant anniversary for every recipient with a functioning graft. Collected data relevant to this study include: patient demographics, transplant date, donor type, graft status, and immunosuppressant medications. The USRDS also houses claims for Medicare Part A (institutional), Part B (physician/supplier), including outpatient immunosuppressant medications, and Part D (prescription drug benefit).

The study sample included all adult RTRs 18 years of age or older who: 1) received their primary renal transplant between July 1, 2006 and December 31, 2011;2) experienced graft survival for at least 12 months posttransplant and had 12 months of data in the USRDS; 3) were enrolled in Medicare Part D and had continuous Medicare coverage; and 4) were prescribed tacrolimus. July 1, 2006 was selected as the start date of the study to allow a 6-month stabilization period to address any problems/confusion in the Part D enrollment process following its implementation in January 2006. December 31, 2012 denotes the end of the study period, as it is the last date available in the Medicare claims data. Data were collected for RTRs from the date of transplant until the end of their first year posttransplant, as the first 12 months posttransplant is an important time period in establishing adherent behaviors. Chisholm et $\mathrm{al}^{15}$ noted that among RTRs who did not receive targeted interventions, IST adherence decreased from $100 \%$ to $<70 \%$ during the first 12 months following transplant. Tacrolimus was selected as the immunosuppressant medication of study because it is the most widely used calcineurin inhibitor.

This study was approved by the University of Tennessee Health Science Center Institutional Review Board.

Adherence to IST was based on Medicare Parts B and D prescription claims data and measured by medication possession ratio (MPR). MPR has been used in previous studies involving the USRDS and Medicare claims and was calculated for each RTR included in the study. ${ }^{1,2}$ MPR was defined as the number of days supplied (ie, number of days an RTR "possessed" IST) divided by 365 days (number of days in the 12-month posttransplant period), excluding days the RTR was hospitalized. The number of days supplied was determined based on the individual RTR's cycle of refills. To explain further, IST prescriptions were classified as 30-, 60-, or 90-day cycles. Each fill was assumed to contain: 1) a 30 -day supply if the number of days between refills was $\leq 45$; 2) a 60-day supply if the number of days between refills was $>45$ but $\leq 75$; or 3 ) a 90 -day supply if the number of days between refills was $>75$. The total number of days supplied was then calculated by adding the estimated days supplied for each refill for all prescriptions filled within the 12-month time period. This method of determining cycle of refills was used in a prior USRDS study. ${ }^{2}$

MTM eligibility was used as a proxy for actual MTM services. Two sets of criteria were used to determine MTM eligibility. The 2006 MTM thresholds established by CMS were used to determine eligibility for RTRs transplanted during the years 2006-2009: two to five chronic disease states, two to 15 Part D-covered drugs, and minimum drug costs of US $\$ 4,000 .{ }^{16,17}$ For RTRs transplanted 2010 and later, the 2010 MTM thresholds revised by CMS were applied: two 
to three chronic disease states, two to eight Part D-covered drugs, and minimum drug costs of US\$3,000. ${ }^{18}$ Medicare claims during the follow-up period were used to determine each RTR's number of chronic conditions, number of Part D drugs used, and annual drug costs. Number of chronic conditions was determined based on ICD-9-CM codes for common disease states of interest identified by $\mathrm{CMS}^{19}$ and Daniel and Malone. ${ }^{20}$ The number of Part D drugs was calculated based on unique prescription fills; this variable was set to 0 if RTRs had no Part D claims. Drug costs were calculated as the sum of gross drug costs for all prescriptions filled by an RTR.

In the main analyses, IST adherence was defined as an MPR of $\geq 0.8$, a value commonly used to distinguish between medication adherence and nonadherence in the literature. . $3,21,22$ Additionally, median threshold levels were used to determine who was or was not eligible for MTM. For RTRs transplanted from 2006 to 2009, the median threshold was eight for the number of Part D drugs and three for chronic conditions. For RTRs transplanted 2010 or later, the median threshold was five for number of Part D drugs and two for chronic conditions. Medicare Part D plans have the flexibility to establish eligibility criteria within the threshold ranges established by CMS. Therefore, sensitivity analyses were also performed using both "low" and "high" threshold criteria. It was expected that more RTRs would be MTMeligible when the "low" thresholds were applied, and fewer RTRs would be eligible when the "high" thresholds were applied. For RTRs transplanted from 2006 to 2009, the "low" thresholds were two Part D drugs and two chronic conditions, while the "high" thresholds were 15 part D drugs and five chronic conditions. For RTRs transplanted in 2010 or later, the "low" thresholds were two Part D drugs and two chronic conditions, while the "high" thresholds were eight Part D drugs and three chronic conditions. An additional sensitivity analysis, in which adherence was indicated as MPR $\geq 0.9$ rather than 0.8 (as 0.9 is commonly used as a secondary or alternative adherence threshold), was also conducted using median MTM eligibility threshold values..$^{21,22}$

\section{Statistical analysis}

Descriptive statistics were estimated using the FREQ and UNIVARIATE procedures of SAS ${ }^{\circledR}$ version 9.3 (SAS Institute Inc., Cary, NC, USA). The LOGISTIC procedure was used to estimate multiple logistic regression models of adherence; odds ratios (ORs) were calculated. Categorical variables included in the full model were sex, race/ethnicity, type of donor, MTM eligibility, and education; continuous variables included in the full model were age in decades, number of prescription drugs, and number of chronic conditions. Variables included in the full model with significance levels $>0.10$ were generally removed from the model, beginning with the variable with the largest $P$-value. The best subsets selection was used to confirm that the reduced model was the most appropriate choice. Sensitivity analyses were performed on the reduced model by changing the definition of the MTM eligibility variable and by changing the threshold for adherence to 0.90 (as previously described). An alpha of 0.05 was used to determine statistical significance.

\section{Results}

Among the 17,181 RTRs included in the study (Figure 1), the sample was predominately male $(59.1 \%)$ and White (40.5\%) (Table 1). At 12 months posttransplant, mean MPR was $0.91 \pm 0.17$ (mean \pm standard deviation), with $16.8 \%$ of participants having an MPR of $<0.80$.

As displayed in Table 2, sex, MTM eligibility, age, and number of prescription drugs were significantly associated $(P<0.05)$ with IST adherence in the full model at 12 months posttransplant, after controlling for all other variables in the model. Specifically, females were more likely than males to

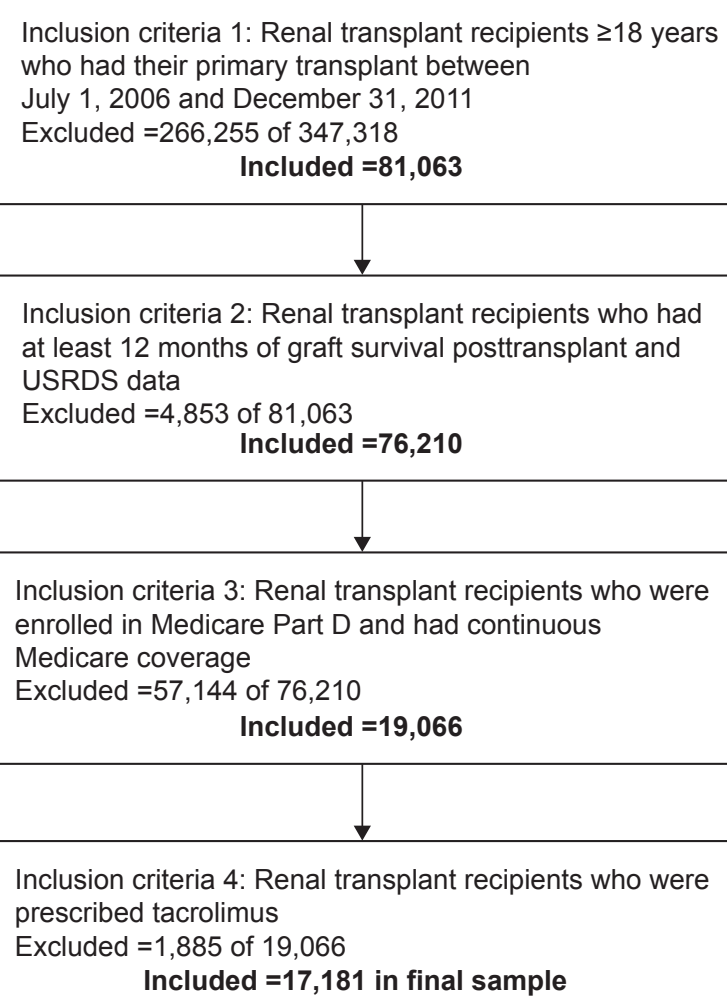

Figure I Renal transplant recipient sample inclusion.

Note: Data retrieved from Medicare Parts A, B, and D claims and transplant followup data reported in the USRDS.

Abbreviation: USRDS, United States Renal Data System. 
Table I Characteristics of adult renal transplant recipients $(n=17,181)$

\begin{tabular}{|c|c|}
\hline Characteristics & \\
\hline Mean age \pm SD & $50.2 \pm 14.3$ \\
\hline Median (IQR) & $51.0(22.0)$ \\
\hline \multicolumn{2}{|l|}{ Sex, n (\%) } \\
\hline Female & $7,021(40.9)$ \\
\hline Male & $10,160(59.1)$ \\
\hline \multicolumn{2}{|l|}{ Eligibility thresholds for MTM, n (\%) } \\
\hline $\begin{array}{l}\text { Median thresholds: five or eight Part D drugs, two or } \\
\text { three chronic conditions, and US } \$ 3,000 \text { or US } \$ 4,000 \\
\text { annualized drug costs }\end{array}$ & $7,259(42.3)$ \\
\hline $\begin{array}{l}\text { "Low" thresholds: two Part D drugs two chronic } \\
\text { conditions, and US } \$ 3,000 \text { or US } \$ 4,000 \text { annualized } \\
\text { drug costs }\end{array}$ & $8,196(47.7)$ \\
\hline $\begin{array}{l}\text { "High" thresholds: eight or I5 Part D drugs, three } \\
\text { or five chronic conditions, and US } \$ 3,000 \text { or US } \$ 4,000 \\
\text { annualized drug costs }\end{array}$ & $3,719(21.7)$ \\
\hline Mean number of prescription medications \pm SD & $9.3 \pm 5.3$ \\
\hline Median (IQR) & $9(6)$ \\
\hline Mean number of chronic conditions \pm SD & $5.8 \pm 2.3$ \\
\hline Median (IQR) & $5(3)$ \\
\hline \multicolumn{2}{|l|}{ Race/ethnicity, n (\%) } \\
\hline White & $6,959(40.5)$ \\
\hline Black & $5,67 \mid(33.0)$ \\
\hline Hispanic $^{\mathrm{a}}$ & $3,332(19.4)$ \\
\hline Other ${ }^{b}$ & $1,219(7.1)$ \\
\hline \multicolumn{2}{|l|}{ Type of transplant, n (\%) } \\
\hline Deceased donor & $12,847(74.8)$ \\
\hline Living donor & $4,334(25.2)$ \\
\hline \multicolumn{2}{|l|}{ Highest education level attained, $\mathrm{n}(\%)$} \\
\hline Unknown or attended grades $0-8^{c}$ & $3,677(21.4)$ \\
\hline Attended high school (grades 9-12) or graduate & $8,007(46.6)$ \\
\hline Attended college or technical school & $3,313(19.3)$ \\
\hline College or postcollege graduate ${ }^{d}$ & $2,184(12.7)$ \\
\hline Nonadherent (MPR <0.80), n (\%) & $2,892(16.8)$ \\
\hline Mean MPR $\pm S D$ & $0.91 \pm 0.17$ \\
\hline Median (IQR) & $1.0(0.2)$ \\
\hline
\end{tabular}

Notes: ${ }^{a}$ Black and Hispanic: $n=155$; Other race and Hispanic: $n=194$; White and Hispanic: $n=2,983$; Hispanic response of yes or no was unknown for 201 observations. 'Native American: $n=209$; Asian: $n=968$; Unknown: $n=7$; Other: $n=35$. 'Grade school (grades 0-8): $n=1,436$; none: $n=153$; unknown: $n=2,087$; missing: $n=1$. ${ }^{d}$ College or postcollege graduate category: Associate or Bachelor degree: $n=1,600$; postcollege graduate degree: $n=584$.

Abbreviations: IQR, interquartile range; MTM, medication therapy management; MPR, medication possession ratio; SD, standard deviation.

be adherent (adjusted OR $=1.19,95 \%$ confidence interval $[\mathrm{CI}]=1.10-1.29, P<0.0001)$. MTM-eligible RTRs were more likely to be adherent than those who were not MTM-eligible (adjusted OR $=1.13,95 \% \mathrm{CI}=1.02-1.26, P=0.02$ ). For every 10 -year increase in age (over 18 years), odds of adherence decreased (adjusted OR $=0.96,95 \% \mathrm{CI}=0.93-0.99, P=0.02$ ). RTRs taking more prescription drugs were more likely to be adherent than those taking fewer prescription drugs (adjusted $\mathrm{OR}=1.01,95 \% \mathrm{CI}=1.003-1.03, P=0.02$ ).

Table 3 displays ORs estimated from the reduced model (main analysis), which was the most appropriate model choice, as well as from sensitivity analyses. In the main analysis (in which MTM eligibility was determined using median threshold values) and the "low" threshold sensitivity analysis (in which MTM eligibility was determined using "low" threshold values), female sex, being MTM-eligible, and taking more prescription medications were associated with increased likelihood of adherence $(P<0.05)$, while incremental increases in age were associated with decreased likelihood of adherence $(P=0.03)$. In the "high" threshold sensitivity analysis (in which MTM eligibility was determined using "high" threshold values), sex, MTM eligibility, and age remained significant $(P<0.05)$, but the number of prescription drugs did not. In the final sensitivity analysis, in which MTM eligibility was determined using median threshold values and adherence criteria of MPR $\geq 0.90$, RTRs who were female $(\mathrm{OR}=1.13,95 \% \mathrm{CI}=1.05-1.21)$, Black $(\mathrm{OR}=1.14,95 \% \mathrm{CI}$ $=1.06-1.23)$, Hispanic $(\mathrm{OR}=1.16,95 \% \mathrm{CI}=1.06-1.27)$, and MTM-eligible ( $\mathrm{OR}=1.12,95 \% \mathrm{CI}=1.02-1.21)$ were more likely to be adherent compared to RTRs who were male, White, and not MTM-eligible, respectively $(P<0.05)$. The results of the sensitivity analyses suggest that as the MTM eligibility threshold is increased, the odds of adherence increase ("low" threshold OR of 1.11 versus "high" threshold OR of 1.36), thereby indicating a greater effect on RTRs with higher threshold values compared with lower ones. In contrast, changing the definition of adherence from MPR $\geq 0.8$ to a more stringent value of $\geq 0.9$ resulted in a relatively small change in the odds of adherence based on MTM eligibility (OR of 1.14 using 0.8 MPR vs OR of 1.12 using 0.9 MPR).

\section{Discussion}

The purpose of this study was to evaluate the effects of Medicare Part D MTM eligibility on IST adherence among adult RTRs at 12 months posttransplant. Previous research has provided evidence that MTM services have a positive effect on medication adherence. ${ }^{7}$ For example, Skinner et a ${ }^{23}$ found that in a sample of patients with poorly controlled diabetes, $62 \%$ of individuals who received MTM were adherent to medication compared to only $7 \%$ in the non-MTM group. The current study is consistent with these findings. At 12 months posttransplant, MTM eligibility was associated with better IST adherence. In fact, MTM-eligible RTRs were 1.14 times more likely to be adherent compared to non-MTM-eligible RTRs. The effects of MTM eligibility remain significant in sensitivity analyses, suggesting a consistently favorable relationship in which MTM-eligible RTRs were 1.11 ("low" thresholds) to 1.36 ("high" thresholds) times more likely to be adherent to IST than those who were not MTM-eligible. 
Table 2 Associations between adherence and RTRs' characteristics at 12 months posttransplant, adjusted odds ratios, and $95 \%$ confidence intervals

\begin{tabular}{llll}
\hline Variable & Adjusted odds ratio & 95\% confidence interval & P-value \\
\hline Female vs male & 1.19 & $1.10-1.29$ & $<0.0001$ \\
Black vs White & 1.10 & $1.00-1.21$ & 0.06 \\
Hispanic vs White & 1.11 & $0.99-1.25$ & 0.07 \\
Other vs White & 1.01 & $0.86-1.19$ & 0.89 \\
Cadaveric vs living & 0.95 & $0.94-1.04$ & 0.27 \\
MTM': yes vs no & 1.13 & $1.02-1.26$ & 0.02 \\
Age (IO years) & 0.96 & $0.93-0.99$ & 0.02 \\
Less than high school vs college degree & 0.99 & $0.86-1.15$ & 0.91 \\
High school vs college degree & 1.00 & $0.88-1.14$ & 0.97 \\
Attended college/technical school vs college degree & 0.90 & $0.78-1.04$ & 0.17 \\
Numbers of prescription drugs & 1.01 & $1.003-1.03$ & 0.02 \\
Numbers of chronic conditions & 1.01 & $0.99-1.03$ & 0.33 \\
\hline
\end{tabular}

Notes: aMTM eligibility determined using median threshold values: for RTRs transplanted from 2006 to 2009 , this was eight Part D drugs and three chronic conditions; for RTRs transplanted 2010 or later, this was five Part D drugs and two chronic conditions. ${ }^{b}$ Age modeled in 10 -year increments beginning at age 18 years.

Abbreviations: MTM, medication therapy management; RTRs, renal transplant recipients.

The results of this study support the basic premise of MTM - that provision of medication management services, such as medication reviews, development of a medicationrelated action plan, and follow-up, will result in improved medication-taking behaviors, including better adherence. ${ }^{10}$
The importance of medication adherence cannot be overemphasized, both in terms of health outcomes and health care costs. Prior studies have noted that medication nonadherence is related to increased morbidity and mortality. ${ }^{24,25}$ Among RTRs, for example, IST nonadherence

Table 3 Associations between adherence and RTRs' characteristics at 12 months posttransplant: adjusted odds ratios and $95 \%$ confidence intervals from the reduced model and sensitivity analyses

\begin{tabular}{|c|c|c|c|}
\hline Model/variable & Adjusted odds ratio & 95\% confidence interval & $P$-value \\
\hline \multicolumn{4}{|l|}{ Main analysis/reduced model ${ }^{a}$} \\
\hline Female vs male & 1.19 & $1.09-1.29$ & $<0.0001$ \\
\hline Black vs White & 1.09 & $0.99-1.20$ & 0.09 \\
\hline Hispanic vs White & 1.12 & $1.00-1.25$ & 0.06 \\
\hline Other vs White & 1.00 & $0.85-1.18$ & 0.99 \\
\hline MTM: yes vs no & 1.14 & $1.02-1.26$ & 0.02 \\
\hline Age $(10 \text { years })^{b}$ & 0.97 & $0.94-0.99$ & 0.03 \\
\hline Numbers of prescription drugs & 1.02 & $1.01-1.03$ & 0.002 \\
\hline \multicolumn{4}{|c|}{ Sensitivity analysis: "Low" thresholdsc } \\
\hline Female vs male & 1.19 & $1.09-1.29$ & $<0.0001$ \\
\hline Black vs White & 1.09 & $0.99-1.20$ & 0.09 \\
\hline Hispanic vs White & 1.12 & $1.00-1.25$ & 0.06 \\
\hline Other vs White & 1.00 & $0.85-1.17$ & 0.99 \\
\hline MTM-low: yes vs no & 1.11 & $1.01-1.22$ & 0.04 \\
\hline Age $(10 \text { years })^{b}$ & 0.97 & $0.94-0.99$ & 0.03 \\
\hline Numbers of prescription drugs & 1.02 & $1.01-1.03$ & 0.0002 \\
\hline \multicolumn{4}{|c|}{ Sensitivity analysis: "High" thresholds ${ }^{d}$} \\
\hline Female vs male & 1.19 & $1.09-1.29$ & $<0.000$ I \\
\hline Black vs White & 1.09 & $0.99-1.20$ & 0.09 \\
\hline Hispanic vs White & 1.12 & $1.00-1.25$ & 0.06 \\
\hline Other vs White & 1.00 & $0.85-1.17$ & 0.99 \\
\hline MTM-high: yes vs no & 1.36 & $1.20-1.56$ & $<0.0001$ \\
\hline Age $(10 \text { years })^{b}$ & 0.97 & $0.94-0.99$ & 0.02 \\
\hline Numbers of prescription drugs & 1.01 & $1.00-1.02$ & 0.06 \\
\hline
\end{tabular}

Notes: aMTM eligibility determined using median threshold values: for RTRs transplanted from 2006 to 2009, this was eight Part D drugs and three chronic conditions; for RTRs transplanted 2010 or later, this was five Part D drugs and two chronic conditions. ${ }^{b}$ Age modeled in 10 -year increments beginning at age 18 years. ${ }^{~}$ MTM eligibility determined using "low" threshold values: for RTRs transplanted from 2006 to 2009, this was two Part D drugs and two chronic conditions; for RTRs transplanted 2010 or later, this was two Part D drugs and two chronic conditions. 'MTM eligibility determined using "high" threshold values: for RTRs transplanted from 2006 to 2009 , this was 15 Part D drugs and five chronic conditions; for RTRs transplanted 2010 or later, this was eight Part D drugs and three chronic conditions.

Abbreviations: MTM, medication therapy management; RTR, renal transplant recipients. 
is a leading cause of preventable graft failure. ${ }^{3}$ It has been estimated that, on an annual basis, medication nonadherence contributes to US\$100 billion in inpatients costs and US\$2,000 per patient in excess physician visits. ${ }^{26}$ Further, a report by the New England Healthcare Institute found that medication nonadherence contributed to avoidable health care spending of approximately US $\$ 290$ billion annually. ${ }^{27}$ The New England Healthcare Institute made several recommendations to improve adherence that are compatible with the MTM model, including conducting medication reviews, addressing cost barriers, engaging patients in care, and providing patients with education regarding their disease state and medication regimen. ${ }^{27}$

Thus, it is evident that MTM may be of great benefit to chronic disease patients, particularly RTRs who take multiple medications per day (IST plus medications to treat comorbidities, such as hypertension, hyperlipidemia, and diabetes). Yet, less than half the RTRs in this study's sample were eligible for MTM under Medicare Part D based on thresholds established by CMS, even when the "low" threshold levels were considered. This suggests the criteria used to determine MTM eligibility may be prohibitive to more widespread utilization of Part D's MTM benefit. Wang et $\mathrm{al}^{28}$ and Wang et $\mathrm{al}^{29}$ have also reported challenges facing the current CMS thresholds, namely, an increased number of Part D plans using the maximum allowable thresholds (which restricts MTM enrollment) as well as persistent racial and ethnic disparities in MTM eligibility. Based on the cumulative evidence, we recommend CMS explore alternative MTM eligibility thresholds which expand rather than limit access to MTM services. Future studies should evaluate these potential alternative thresholds and their effects on MTM eligibility.

Other variables related to adherence in this study included the number of prescription drugs, sex, and age. Specifically, taking a greater number of prescription medications resulted in increased adherence. This finding conflicts with previous research which noted decreased adherence as a result of increased number of medications or medication doses in RTRs. ${ }^{30}$ We speculate that RTRs who take additional prescription drugs to treat comorbid conditions may be more adherent to IST because it is integrated into an established medication regimen and routine. Those who take fewer prescription medications may be less likely to develop habitual medication-taking behaviors. Therefore, intervention efforts should focus on assisting RTRs in developing patterns of behavior supportive of maintaining medication adherence. Other variables associated with adherence, sex and age, are not modifiable, and as such are more difficult intervention targets. However, they do suggest individuals who may need more adherence support during the first year posttransplant - males and older adults.

\section{Limitations}

There are limitations to this study. The use of claims records as a measure of IST adherence may be limited as they indicate medication "possession", not medication "use". However, claims records are generally considered an acceptable proxy of medication use, and Medicare claims records have been used successfully to determine MPR in prior published studies examining IST adherence. ${ }^{1,2}$ Another limitation is that because the study is retrospective in nature, a causal relationship between MTM and IST adherence cannot be established. Although the evidence found in this study supports that odds of adherence increase when RTRs are MTM-eligible, prospective studies are needed to more comprehensively examine the causal effects of MTM eligibility on IST adherence among RTRs. Such studies may include a time-series analysis comparing the IST adherence of RTRs who receive MTM as part of their Part D benefits versus RTRs who were not eligible for MTM, or a pre- and poststudy comparing the adherence of RTRs before and after exposure to Part D MTM services.

\section{Conclusion}

The study found that at 12 months posttransplant, RTRs who were MTM-eligible under Medicare Part D were more likely to be IST adherent than those RTRs who were not MTM-eligible. However, less than half of the included RTRs were MTM-eligible, suggesting CMS thresholds may need to be reconsidered to increase access and, in turn, improve medication adherence in this patient population.

\section{Acknowledgments}

We would like to acknowledge Emanuel Villa Baca and Ian Brooks at the University of Tennessee Health Science Center for their assistance with this project. The data reported here have been supplied by the USRDS. The interpretation and reporting of these data are the responsibility of the authors and in no way should be seen as an official policy or interpretation of the US government. The results presented in this paper have not been published previously in whole or part.

\section{Disclosure}

The authors report no conflicts of interest in this work. 


\section{References}

1. Pinsky BW, Takemoto SK, Lentine KL, Burroughs TE, Schnitzler MA, Salvalaggio PR. Transplant outcomes and economic costs associated with patient noncompliance to immunosuppression. Am J Transplant. 2009;9:2597-2606.

2. Spivey CA, Chisholm-Burns MA, Damadzadeh B, Billheimer D. Determining the effect of immunosuppressant adherence on graft failure risk among renal transplant recipients. Clin Transplant. 2014;28: 96-104.

3. Butler JA, Roderick P, Mullee M, Mason JC, Peveler RC. Frequency and impact of nonadherence to immunosuppressants after renal transplantation: a systematic review. Transplantation. 2004;77:769-776.

4. Denhaerynck K, Dobbels F, Cleemput I, et al. Prevalence, consequences, and determinants of nonadherence in adult renal transplant patients: a literature review. Transpl Int. 2005;18:1121-1133.

5. Chisholm MA, Lance CE, Williamson GM, Mulloy LL. Development and validation of an immunosuppressant therapy adherence barrier instrument. Nephrol Dial Transplant. 2005;20:181-188.

6. Chisholm MA, Mulloy LL, DiPiro JT. Comparing renal transplant patients' adherence to free cyclosporine and free tacrolimus immunosuppressant therapy. Clin Transplant. 2005;19:77-82.

7. Viswanathan M, Kahwati LC, Golin CE, et al. Medication therapy management interventions in outpatient settings: a systematic review and meta-analysis. JAMA Intern Med. 2015;175:76-87.

8. Wang J, Qiao Y, Shih Y-CT, et al. Effects of Medicare Part D on disparity implications of medication therapy management eligibility criteria. Am Health Drug Benefits. 2014;7(6):346-358.

9. American Pharmacists Association. APhA MTM Central. What is medication therapy management? Available from: http://www.pharmacist. com/mtm. Accessed August 19, 2015.

10. Medication Therapy Management in Pharmacy Practice: Core Elements of an MTM Service Model. Version 2.0; 2008. Available from: http:// www.pharmacist.com/sites/default/files/files/core_elements_of_an_ mtm_practice.pdf. Accessed November 17, 2015.

11. Thumar R, Zaiken K. Impact of live medication therapy management on cholesterol values in patients with cardiovascular disease. J Am Pharm Assoc. 2014;54:526-529.

12. Planas LG, Crosby KM, Mitchell KD, Farmer KC. Evaluation of a hypertension medication therapy management program in patients with diabetes. J Am Pharm Assoc. 2009;49:164-170.

13. Hirsch JD, Gonzales M, Rosenquist A, Miller TA, Gilmer TP, Best BM Antiretroviral therapy adherence, medication use, and health care costs during 3 years of a community pharmacy medication therapy management program for Medi-Cal beneficiaries with HIV/AIDS. J Manag Care Pharm. 2011;17(3):213-223.

14. Moore JM, Shartle D, Faudskar L, Matlin OS, Brennan TA. Impact of a patient-centered pharmacy program and intervention in a high-risk group. J Manag Care Pharm. 2013;19(3):228-236.

15. Chisholm MA, Mulloy LL, Jagadeesan M, DiPiro JT. Impact of clinical pharmacy services on renal transplant patients' compliance with immunosuppressive medications. Clin Transplant. 2001;15: 330-336.

16. Centers of Medicare and Medicaid Services. Medication therapy management. Available from: https://www.cms.gov/Medicare/PrescriptionDrug-Coverage/PrescriptionDrugCovContra/MTM.html. Accessed August 17, 2015.
17. Medicare Part D Medication Therapy Management (MTM) Programs 2009 Fact Sheet; 2009. Available from: https://www.cms.gov/Medicare/ Prescription-Drug-Coverage/PrescriptionDrugCovContra/downloads/ MTMFactSheet_2009_06-2009_fnl.pdf. Accessed August 17, 2015.

18. 2010 Medicare Part D Medication Therapy Management (MTM) Programs. Fact sheet; 2010. Available from: https://www.cms.gov/Medicare/ Prescription-Drug-Coverage/PrescriptionDrugCovContra/downloads/ MTMFactSheet_2010_06-2010_final.pdf. Accessed August 17, 2015.

19. Centers for Medicare and Medicaid Services. Chronic conditions. Available from: https://www.cms.gov/Research-Statistics-Data-andSystems/Statistics-Trends-and-Reports/Chronic-Conditions/CC_Main. html. Accessed August 19, 2015.

20. Daniel GW, Malone DC. Characteristics of older adults who meet the annual prescription drug expenditure threshold for Medicare medication therapy management programs. J Manag Care Pharm. 2007;13(2): $142-154$.

21. Watanabe JH, Kazerooni R, Bounthavong M. Association of copayment with likelihood and level of adherence in new users of statins: a retrospective cohort study. J Manag Care Pharm. 2014;20: 43-50.

22. Watanabe JH, Bounthavong M, Chen T, Ney JP. Association of polypharmacy and statin new-user adherence in a Veterans Health Administration population: a retrospective cohort study. Ann Pharmacother. 2013;47:1253-1259.

23. Skinner JS, Poe B, Hopper R, Boyer A, Wilkins CH. Assessing the effectiveness of pharmacist-directed medication therapy management in improving diabetes outcomes in patients with poorly controlled diabetes. Diabetes Educ. 2015;41:459-465.

24. Ho PM, Magid DJ, Masoudi FA, McClure DL, Rumsfeld JS. Adherence to cardioprotective medications and mortality among patients with diabetes and ischemic heart disease. BMC Cardiovasc Disord. 2006; 6:48.

25. Sokol MC, McGuigan KA, Verbrugge RR, Epstein RS. Impact of medication adherence on hospitalization risk and healthcare cost. Med Care. 2005;43(6):521-530.

26. Osterberg L, Blaschke T. Adherence to medication. NEngl J Med. 2005; 353(5):487-497.

27. New England Healthcare Institute. Thinking outside the pillbox. A system-wide approach to improving patient medication adherence for chronic disease. New England Healthcare Institute Research Brief; 2009. Available from: http://www.nehi.net/publications/17thinking-outside-the-pillbox-a-system-wide-approach-to-improvingpatient-medication-adherence-for-chronic-disease/view. Accessed November 17, 2015.

28. Wang J, Shih YC, Qin Y, et al. Trends in Medicare Part D medication therapy management eligibility criteria. Am Health Drug Benefits. 2015; $8(5): 247-255$.

29. Wang J, Zhang Z, Spivey CA, Chisholm-Burns M. Historical trend of racial and ethnic disparities in meeting Medicare medication therapy management eligibility in non-Medicare population. Res Social Adm Pharm. 2014;10:904-917.

30. Kuypers DR, Peeters PC, Sennesael JJ, et al. Improved adherence to tacrolimus once-daily formulation in renal recipients: a randomized controlled trial using electronic monitoring. Transplantation. 2013;95: $333-340$.
Patient Preference and Adherence

\section{Publish your work in this journal}

Patient Preference and Adherence is an international, peer-reviewed, open access journal that focuses on the growing importance of patient preference and adherence throughout the therapeutic continuum. Patient satisfaction, acceptability, quality of life, compliance, persistence and their role in developing new therapeutic modalities and compounds to optimize

\section{Dovepress}

clinical outcomes for existing disease states are major areas of interest for the journal. This journal has been accepted for indexing on PubMed Central. The manuscript management system is completely online and includes a very quick and fair peer-review system, which is all easy to use. Visit http://www. dovepress.com/testimonials.php to read real quotes from published authors. 